Results 26 patients were identified based on the keyword search. 6/26 patients (19 months - 15 years) fulfilled the diagnostic criteria of anaphylaxis; however, only $2 / 6$ had clearly documented diagnosis of anaphylaxis. All 6 were treated with Chlorphenamine; 4/6 had IM adrenaline; and 4/ 6 had IV Hydrocortisone. 3/6 patients did not have an identifiable allergen trigger, all of whom did not have a tryptase level taken. 5/6 had minimum recommended time of 6 hours of observation. At discharge, 4/6 patients had an auto-injector prescribed and training provided. All patients had documented safety-advice given, with an anaphylaxis action plan provided to $4 / 6$. Overall, specific documentation pertaining to information on discharge, including risk of biphasic reaction (1/6), was poor. Reassuringly, all patients were referred to the allergy clinic.

Conclusions This audit identifies the need for further education on recognition, correct diagnosis and management of anaphylaxis, with emphasis on clear documentation especially of safety-net information given at discharge. Promoting use of the Anaphylaxis Proforma and guideline aims to improve safety and standardise the care of patients presenting with anaphylaxis.

\section{G585(P) SUPERVISED FEEDS: A REVIEW OF LOCAL PRACTICE}

CE Baker, A Aston, N Patel. Paediatric Allergy, Royal London Hospital, Barts Health NHS Trust, London, UK

\subsection{6/archdischild-2020-rcpch.502}

Background Food allergy is becoming increasingly common in the paediatric population resulting in increased demand for allergy services. Food allergy investigations can include inhospital oral food challenges and supervised feeds. In 2018 supervised feeds were introduced in this hospital for challenges deemed as lower risk. This involves consuming a predetermined amount of food within 30 minutes and being observed for an hour thereafter. An oral food challenge involves food given in incremental doses with observations in-between and 2 hours after, therefore, it is a longer process.

Aims To review current departmental practice of supervised feeds focussing on the following:

1. What patterns of food are being tested?

2. What were the skin prick test (SPT)/specific IgE results?

3. Explore test failures in further detail.

Method A retrospective analysis of supervised feeds recorded in the unit database from May 2018 to May 2019. If data was missing then discharge summaries from the admission were reviewed.

Results The number of supervised feeds recorded on the database between May 2018-2019 totalled 162, 156 of these were included. The most common foods tested were tree nuts $(30 \%)$ followed by peanuts (20\%). SPT results ranged from 0-4 mm. 2 patients' had results higher than recommended by current guidelines, however, both passed their supervised feed. Overall failure rate was $13.5 \%$. SPT was 0 $\mathrm{mm}$ in $64 \%$ of patients and $11 \%$ of these failed. Reasons for failure included refusal to eat (4 patients) and allergic reaction; ranging from itching to anaphylaxis (1 patient). Treatment required ranged from observation, antihistamine, to IM adrenaline (1patient).
Conclusion Supervised feeds are a useful method to assessing food allergy in appropriately selected patients. Overall failure rate of supervised feeds in this study was low at $13.5 \% .10$ patients had a SPT of $0 \mathrm{~mm}$ and failed, so it is important to remember even though deemed low risk there is still a risk of significant reaction. By identifying appropriate patients as suitable for supervised feed then their time spent in hospital can be reduced and may allow for an increased number of in-hospital challenges throughout the year.

\section{G586(P) CASE REPORT OF HYPER IGM SYNDROME IN DOWN SYNDROME CHILD}

MC Quek, A Migowa, RW Gitakah. Department of Paediatrics and Child Health, Aga Khan University Hospital, Nairobi, Kenya

\subsection{6/archdischild-2020-rcpch.503}

Introduction Down syndrome is the most common genetic disease that often presents with a high frequency of infections especially upper airway infections in their early years, characterized by increased severity and prolonged course of disease. Individuals with Down syndrome are known to have abnormal function of the B-cell function, which might present with high $\mathrm{IgG}$ and low $\operatorname{IgM}$ serum level.

Case Description A one-year-old known Down syndrome child presented with persistent nasal congestion, fever, noisy breathing, and daily cough for 8 months. He had several outpatient consults and treatment with antibiotics but with poor relief of symptoms. Due to persistent symptoms, he was referred to the ENT surgeon. His paranasal radiographs revealed sinusitis. He was then referred to a pediatric infectious specialist for further management. Examination revealed a sick-looking, syndromic child who was having marked nasal congestion, global hypotonia and generalized erythematous rash. He was well-nourished. Serum immunoglobulin test show marked elevated Ig $\mathrm{M}$ with low Ig G and Ig A levels. Rheumatologist agreed with the possible diagnosis of primary immunodeficiency syndrome (PIDS). Pulmonologist confirmed no underlying congenital lung pathology from the chest CT scan. The echocardiogram was unremarkable. He received intravenous immunoglobulin (IVIG) as part of the therapy and his symptoms improved markedly. An impression of Hyper Ig $M$ syndrome was made. He received two other courses of IVIG after discharge. Currently, the mother reports good improvement of symptoms.

Discussion Hyper IgM syndrome is a primary immunodeficiency syndrome characterized by defective CD 40 signaling, which leads to a defect in Ig class switching recombination.

The association of Down syndrome with PIDS is not clearly demonstrated. In our case, elevated Ig $\mathrm{M}$ was an incidental finding. Warning signs present in children with Down syndrome warrants a detailed immunology investigation to exclude primary immunodeficiency. Diagnosing PIDS imposed a great challenge to doctors especially due to the co-existing condition. A delay in diagnosis is common and associated with increased morbidity and parental distress.

Conclusion There is a need to create awareness amongst the healthcare workers concerning PIDS especially in children with co-existing diseases. We need to assure the proper diagnosis and proper care management are provided to the affected children. 